\title{
Caracterización del nervio digital palmar en el miembro anterior equino
}

\section{Characterization of the palmar digital nerve of the equine forelimb}

\author{
Carolina Ortiz ${ }^{\mathrm{a}}$; Patricio Rodríguez ${ }^{\mathrm{b}}$; Felipe Corrêa $\mathrm{c}$ d*

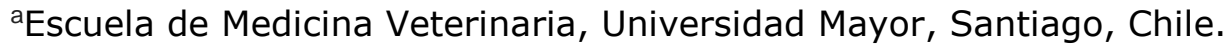

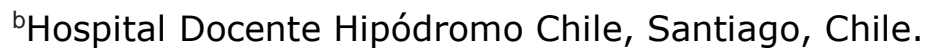 \\ 'Escuela de Medicina Veterinaria, Facultad de Ecología y Recursos Naturales, \\ Universidad Andrés Bello, República 440, Santiago, Chile. \\ dPrograma de Doctorado en Medicina Veterinaria, Facultad de Ecología y Recursos \\ Naturales, Universidad Andrés Bello.
}

*Autor de correspondencia: felipe2983@gmail.com

Recibido: 02 de febrero de 2017; Aceptado: 04 de abril de 2017.

\section{RESUMEN}

A partir de 60 miembros anteriores de caballos mestizos recolectados de un matadero en Santiago, Chile, se determinó que lo más frecuente es que el nervio digital palmar emita sus ramas dorsal y palmar antes (33\%) o al comienzo de la articulación metacarpofalángica (33\%). En un $60 \%$ de los nervios analizados se evidenció la presencia de una a cuatro ramas accesorias y que ésta se presenta de manera similar entre diversas zonas, siendo la presentación más frecuente $(26 \%)$ en la zona de la articulación interfalángica proximal y que se dirigen hacia dorsal. En un $46,66 \%$ de las muestras el nervio digital palmar no se relaciona íntimamente con alguna estructura, seguido con 33,33\% por la unión de éste al ligamento del espolón. Es muy poco frecuente que el nervio emita ramas superficiales que se dirijan hacia la dermis $(6,66 \%)$, que se divida en tres grandes ramas en la articulación metacarpofalángica $(3,33 \%)$ o que pase bajo la arteria digital $(3,33 \%)$, y más frecuente es que éste se disponga sobre la arteria en algún lugar de su recorrido, hecho que ocurrió en el $35 \%$ de los casos. Estos resultados se presentan de manera relevante de considerar en procedimientos diagnósticos o quirúrgicos en la zona distal del miembro anterior del equino, ya que pueden alterar su sensibilidad y eficacia.

Palabras clave: Equino; nervio digital palmar, ramas accesorias. 


\section{ABSTRACT}

60 mixed breed horse forelimb from a slaughterhouse in Santiago, Chile were analyzed. The digital palmar nerve expresses its dorsal and palmar branches before $(33 \%)$ or in the beginning of the fetlock joint (33\%). It was found that $60 \%$ of the samples had between 1 to 4 accessory branches which are presented in a similar way on different areas, being the most frequent (26\%) those located in the pastern joint and aimed to dorsal. In a $46,66 \%$ of the samples the digital palmar nerve is not directly related with any structure, followed in a 33,33\%, by the union of this with the ergot ligament. It is very rare that the nerve expresses superficial branches aimed to the dermis $(6,66 \%)$, divided in three big branches on the fetlock joint $(3,33 \%)$, or went under the digital artery $(3,33 \%)$, and it is more frequent that the nerve went over the artery in some moment of its path, which occurred in the $35 \%$ of the cases. These results are presented in a relevant way, being considered in diagnostic of surgical procedures on the equine forelimb distal region, because they can change its sensibility and effectiveness.

Key words: Equine, digital palmar nerve, accessory branches.

\section{INTRODUCCIÓN}

Se ha descrito que cerca del $50 \%$ de los equinos pueden presentar de una a cinco ramas accesorias que emergen desde las ramas dorsal y palmar del nervio digital palmar (Baxter, 2011; Ross y Dyson, 2011; Mair et al., 2013). Esta variabilidad puede afectar el resultado de procedimientos diagnósticos y quirúrgicos que involucran la zona baja de los miembros anteriores (Floyd et al., 2007, Furst et al., 2012). Es importante la visualización de estas ramas accesorias en neurectomía, por ejemplo, de manera de generar la completa desensibilización de la zona (Ross, 2011; Escobar et al., 2008), evitar complicaciones generados por la íntima relación que tienen con vasos sanguíneos en parte de su recorrido (Furst y Lischer; 2012), o evitar errores en bloqueos neurales, procedimiento utilizado como método diagnóstico (Floyd y Mansmann, 2007), ya que se considera que para una ejecución e interpretación apropiada se requiere un conocimiento adecuado de la anatomía neural implicada (Floyd y Mansmann, 2007; Moyer et al., 2011).

El objetivo del presente estudio fue determinar la variabilidad del nervio digital palmar lateral y medial, estableciendo el número de ramas accesorias que se presentan, su ubicación y relación con la vena, arteria y ligamento del espolón en caballos en Chile.

\section{MATERIAL Y MÉTODOS}

En el estudio se utilizaron 60 miembros anteriores de caballos mestizos y de tamaños similares, recolectados de un matadero en Santiago, Chile. De la totalidad de los miembros, 30 correspondían a miembros anteriores izquierdos (MAI) y 30 miembros anteriores derechos (MAD) que se extendían desde carpo hasta casco. Mediante pre muestreo se estableció que tanto las medidas del nervio y la cantidad de ramas accesorias no variaban entre los nervios digitales palmares laterales y mediales. 
Cada pieza anatómica fue individualmente analizada el mismo día de su faenamiento (para efectos de retracción de tejidos), y posterior a la fijación del nervio la información fue recolectada del nervio digital palmar lateral de cada extremidad. Se determinó: el largo del nervio digital palmar lateral desde el comienzo de los huesos metacarpianos hasta el rodete coronario del casco, medición del diámetro en tres partes de su recorrido (metacarpianos, articulación metacarpofalángica y rodete coronario), identificación de ramas accesorias (número y ubicación), lugar de división del nervio en sus ramas dorsal y palmar, y unión del nervio con estructuras cercanas como vasos sanguíneos y ligamento del espolón. Para situar de manera práctica el lugar donde se ubicaron de las ramas accesorias se dividió la zona estudiada en tres niveles, estableciendo los criterios; alto (si se ubicaron en la zona de la articulación metacarpofalángica), medio (si se presentaban desde el área bajo la articulación metacarpofalángica hasta la articulación interfalángica proximal) y bajo (si se localizaron en zona comprendida desde la articulación interfalángica proximal hasta el rodete coronario). Los resultados se agruparon según las medidas del nervio (largo y ancho), lugar de división del nervio digital en sus ramas dorsal y palmar, ramas accesorias (número y ubicación), y su relación con estructuras cercanas.

La información fue expresada a través de estadística descriptiva y graficada utilizando el programa Excel.

\section{RESULTADOS}

Medidas del nervio digital palmar lateral: la longitud media del nervio digital palmar lateral desde la articulación carpo-metacarpiana hasta su llegada al casco fue similar entre el lado izquierdo $(32,28 \mathrm{~cm})$ y el derecho $(32,49 \mathrm{~cm})$. Además, el mayor diámetro del nervio correspondió a la zona metacarpo-falángica $(3,4 \mathrm{~mm})$, mientras que el de menor diámetro fue en su llegada al casco $(1,7 \mathrm{~mm})$.

Lugar de división del nervio digital palmar en sus ramas dorsal y palmar: Lo más frecuente es que el nervio digital palmar lateral emita sus ramas dorsal y palmar antes (33\%) o al comienzo de la articulación metacarpofalángica (33\%) y menos frecuente su división a medida que avanza hacia distal (Tabla 1).

Tabla 1: Lugar de división del nervio digital palmar lateral según la articulación metacarpofalángica (MTF).

\begin{tabular}{ccc}
\hline $\begin{array}{c}\text { Lugar de división del nervio digital } \\
\text { palmar lateral }\end{array}$ & Número & $\begin{array}{c}\text { Frecuencia de } \\
\text { presentación }\end{array}$ \\
\hline Proximal a la articulación MTF & 20 & $33,33 \%$ \\
Borde proximal de la articulación MTF & 20 & $33,33 \%$ \\
Mitad de la articulación MTF & 13 & $21,66 \%$ \\
Borde distal de la articulación MTF & 5 & $8,33 \%$ \\
Distal a la articulación MTF & 2 & $3,33 \%$ \\
\hline Total & 60 & $100,00 \%$ \\
\hline
\end{tabular}


Número y ubicación de ramas accesorias: en un $48 \%$ de los nervios digitales palmares analizados se evidenció la presencia de una rama accesoria, seguido por aquellos que no presentaban ninguna (40\%). La presentación de dos, tres o cuatro ramas fue poco frecuente llegando a representar el $10 \%$ del total de las muestras (Tabla 2).

Tabla 2: Frecuencia de presentación de ramas accesorias del nervio digital palmar.

\begin{tabular}{ccc}
\hline $\begin{array}{c}\text { Número de ramas } \\
\text { accesorias }\end{array}$ & $\begin{array}{c}\text { Número de } \\
\text { muestras }\end{array}$ & $\begin{array}{c}\text { Frecuencia de } \\
\text { presentación }\end{array}$ \\
\hline 0 & 24 & $40,0 \%$ \\
1 & 29 & $48,3 \%$ \\
2 & 5 & $8,3 \%$ \\
3 & 1 & $1,7 \%$ \\
4 & 1 & $1,7 \%$ \\
Total & 60 & $100,0 \%$ \\
\hline
\end{tabular}

Con respecto al lugar anatómico en donde se genera la rama accesoria, esta se presenta de manera similar entre diversas zonas, siendo la presentación más frecuente (26\%) en la zona de la articulación interfalángica proximal y que se dirigen hacia dorsal (Tabla 3).

Tabla 3: Frecuencia de presentación de ramas accesorias del nervio digital palmar de acuerdo a la zona en donde se generan.

\begin{tabular}{ccc}
\hline $\begin{array}{c}\text { Lugar anatómico de las ramas } \\
\text { accesorias }\end{array}$ & Número & $\begin{array}{c}\text { Frecuencia de } \\
\text { presentación }\end{array}$ \\
\hline Palmar Alto & 9 & $15,00 \%$ \\
Palmar Medio & 7 & $11,67 \%$ \\
Palmar Bajo & 11 & $18,33 \%$ \\
Dorsal Alto & 7 & $11,67 \%$ \\
Dorsal Medio & 16 & $26,67 \%$ \\
Dorsal Bajo & 10 & $16,67 \%$ \\
\hline Total & 60 & $100,00 \%$ \\
\hline
\end{tabular}

Relación del nervio digital palmar lateral con estructuras cercanas: en un $46,66 \%$ de las muestras el nervio digital palmar lateral no se relaciona íntimamente (unión o perforación) con la vena digital, arteria digital ni con ligamentos, seguido con $33,33 \%$ por la unión de éste al ligamento del espolón. Se pesquisó también la unión del nervio con vasos sanguíneos y con otras ramas del mismo, pero fue un hecho poco frecuente (Tabla 4). 
Es muy frecuente que el nervio se disponga sobre la arteria en algún lugar de su recorrido, hecho que ocurrió en el $35 \%$ de los casos. Mientras que rara vez el nervio emite ramas superficiales que se dirijan hacia la dermis $(6,66 \%)$, que se divida en tres grandes ramas en la articulación metacarpofalángica $(3,33 \%)$ o que pase bajo la arteria digital $(3,33 \%)$ (Tabla 5$)$.

Tabla 4: Unión del nervio o sus ramas accesorias con estructuras cercanas.

\begin{tabular}{lcc}
\hline Característica & Cantidad & \multicolumn{2}{c}{ Proporción } \\
\hline Se une al ligamento & 20 & $33,33 \%$ \\
Se une a la vena & 3 & $5,00 \%$ \\
Se une a la arteria & 1 & $1,66 \%$ \\
Se une a la misma rama originaria & 3 & $5,00 \%$ \\
Se une a la rama opuesta & 5 & $8,33 \%$ \\
Sin unión a estructuras & 28 & $46,66 \%$ \\
\hline Total general & 60 & $100,00 \%$ \\
\hline
\end{tabular}

Tabla 5: Frecuencia de otras características del nervio digital palmar.

\begin{tabular}{lcc}
\hline Otras características & Cantidad & Porcentaje \\
\hline Bajo la arteria & 2 & $3,33 \%$ \\
Sobre la arteria & 21 & $35,00 \%$ \\
Rama superficial & 4 & $6,66 \%$ \\
Nervio se divide en 3 & 2 & $3,33 \%$ \\
\hline Total general & 60 & $100,00 \%$ \\
\hline
\end{tabular}




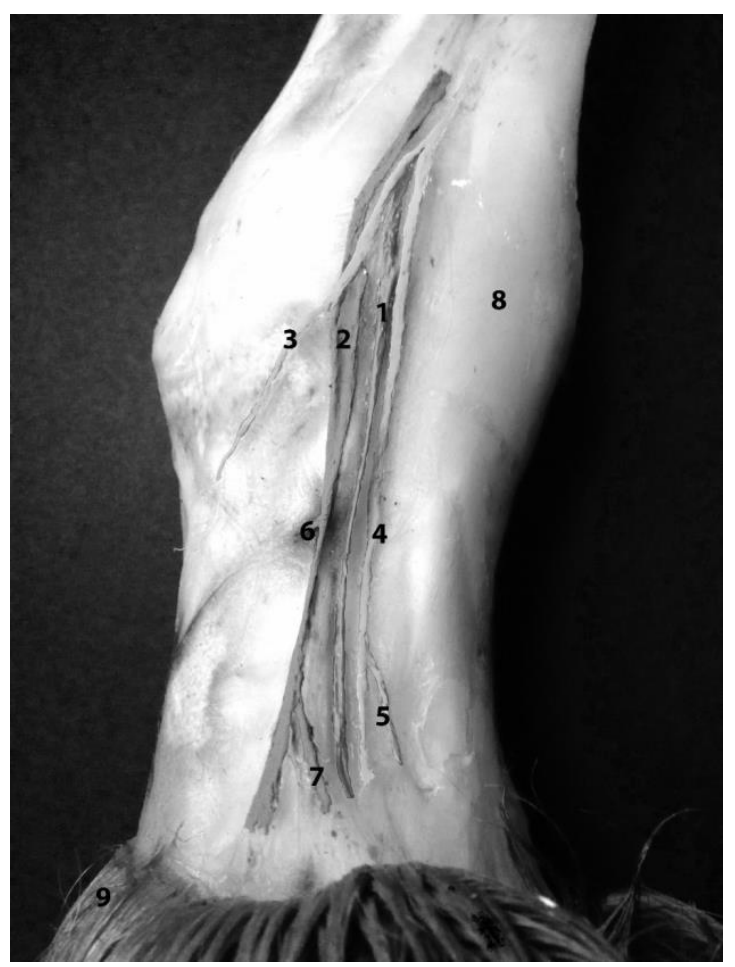

Figura 1. La arteria (1) desciende recta, a diferencia de la vena (2) la cual se ramifica. El nervio palmar emite sus ramas dorsal (3) y palmar (4) al comienzo de la articulación metacarpofalángica. A nivel de la articulación interfalángica proximal emerge una rama accesoria de ubicación palmar (5). La vena se ramifica en la mitad de la primera falange, en una rama que se dirige hacia dorsal (6) y en otra palmar (7) en la parte más distal de la extremidad. Como referencia se pueden observar la articulación metacarpo-falángica (8) y la zona dorso distal de la extremidad (9).

\section{DISCUSIÓN}

Los resultados obtenidos mostraron marcadas diferencias existentes en el recorrido del nervio digital palmar, con presencia de múltiples ramas accesorias en una amplia zona de distribución. Se encontraron evidentes diferencias entre las muestras, hecho que concuerda con otros reportes en la literatura (Baxter, 2011; Ross y Dyson, 2011).

Autores como Mair et al., (2013) y Ross y Dyson (2011) han descrito que en $30 \%$ de los casos una rama intermedia se origina dorsal al nervio, y que en un $50 \%$ continúa sin ramificarse hacia distal, concordando con los resultados obtenidos, donde en un $45 \%$ de las muestras se presentaron ramas dorsales, mientras que en el $40 \%$ continuó hacia distal sin bifurcaciones.

Se observó que el nervio digital palmar puede emitir ramas accesorias las cuales vuelven a unirse distalmente a la misma que le dio origen o a su rama opuesta, hecho relevante en procedimientos como bloqueo anestésico, donde el fármaco administrado puede distribuirse hacia la zona palmar, y a causa de esta unión, interferir con el resultado que se busca (Moreira et al., 1997; Moyer et al. 2011), sin embargo, la información es limitada en cuanto a la propagación de la anestesia local 
en este tipo de analgesia (Nagy et al., 2010), por lo tanto, se considera que las interacciones entre los nervios y las estructuras cercanas deben ser analizadas en profundidad.

Se han descrito variaciones respecto de la descripción anatómica clásica de los nervios palmares en $1 / 3$ de los casos (Baxter, 2011), en el 50\% (Ross y Santana, 2000), e inclusive hasta en más de la mitad de los equinos (Tigero, 2008). Información concordante con los resultados obtenidos, ya que en un $60 \%$ de los casos se pesquisaron ramas accesorias.

En relación al número de ramas accesorias que se presentan en la zona se establecen que pueden ser desde dos a cuatro (Baxter, 2011) o hasta cinco (Ross y Dyson, 2011). Los resultados obtenidos concuerdan con el primer autor, ya que se pesquisaron hasta 4 ramas. Es relevante mencionar que en dos casos se evidenciaron múltiples ramas de pequeño calibre, donde pudiesen existir más de 4 ramas. Estas ramas no fueron consideradas completamente formadas, ya que el grosor no alcanzaba la media de las demás muestras, el cual se acerca a los $1,8 \mathrm{~mm}$.

Entre más proximal el diámetro del nervio es mayor y se ubica más profundo hacia los tejidos (Mair et al. 2013; Cruz, 2003), información que fue corroborada a través de la medición del ancho del nervio en tres partes de su recorrido. Según los resultados expuestos, a medida que el nervio desciende hacia el casco, su diámetro va disminuyendo, al igual que las ramas accesorias que emergen de él.

Se ha descrito que ventral al carpo el nervio emite una rama profunda hacia el ligamento suspensorio y el músculo interóseo, bifurcándose también hacia la piel (Antmann, 2006), hecho concordante con los resultados. Se evidenció, a nivel de la articulación metacarpofalágica, la emisión de tres ramas nerviosas, a diferencia de dos, donde la tercera rama, de ubicación más palmar, se dirigía hacia la piel. Se sugiere que puede ser esta misma rama que en ocasiones se dirija a inervar tanto a ligamento suspensorio, como al interóseo.

Los resultados obtenidos concuerdan con que las variaciones en los nervios digitales son tan comunes que, en realidad, no se puede describir una anatomía definitiva (Baxter, 2011, Santana y Concha 2000), ya que dichas ramas fueron pesquisadas en toda el área distal del miembro anterior, no repitiéndose ninguno de manera significativa.

\section{AGRADECIMIENTOS}

Al personal del Hospital Docente Hipódromo Chile, y Matadero Lo Blanco por proporcionar las muestras. 


\section{REFERENCIAS}

Amtmann, G. 2006. Anatomía del Equino, Canino, Porcino y Aves. 5ta ed. Universidad Mayor. Chile, Domus S.A . Lillo, Selma.

Baxter, G. 2011. Adams and Stashk's Lameness in Horses. 6 ed., UK. Blackwell Publishing.

Budras, K., Sack, W., y Rock, S. 2009. Anatomy of the horse. 5 ed. Alemania, Schlutersche Verlagsgesellschaft.

Cruz, J. 2003. Bloqueo sesamoideo abaxial con ketamina en la caballo. Universidad de Complutense, Madrid. Javier López San Román.

Floyd, A, y Mansmann, R. 2007. Equine Podiatry. 1 ed, USA. Elsevier Health Science.

Furst, A, y Lischer, C. 2012. Equine Surgery. 4 ed. Estados Unidos, Elsevier.

Mair, T., Love, S., Schumacher, J., y Smith, R. 2013. Frazer, G. Equine Medicine, Surgery and Reproduction. 2 ed, Estados Unidos, Elsevier Ltd.

Moreira, E. 1997. Ciencia Rural. Estudio Anatómico de la Inervación de la Porción Distal del Miembro Torácico del Equino. Revista Ciencia Rural. 27(1).

Moyer, W., Schumacher, J., y Schumacher, J. 2011. Equine Joint Injection and Regional Anesthesia. 2da ed, Estados Unidos. Academy Veterinary Solutions.

Ross, M., y Dyson, S. 2011. Diagnosis and Managment of Lameness in the Horse. 2 ed., Estados Unidos. Elsevier Saunders.

Santana, R., y Concha, I. 2000. Características Morfológicas del Nervio Digital Palmar en Equinos Mestizos. Universidad Santo Tomás, Chile. Alberto Rodríguez.

Shultz, L. 2004. Howell Equine Handbook of Tendon and Ligament Injuries. 1 ed, New Jersey Wiley.

Verheyen, K. 2004. Descriptive Epidemiology of Fractures Occurring in British Thoroughbred Racehorses in Training. Equine Veterinary Journal, 36:167-173. 